\title{
DIFFERENT METHODS OF FORMING COLD FRONTS IN NON-MERGING CLUSTERS
}

\author{
RENATO DUPKE \\ University of Michigan, Ann Arbor \\ RAYMOND E. WHITE III \\ University of Alabama, Tuscaloosa \\ JOEL N. BREGMAN \\ University of Michigan, Ann Arbor \\ Draft version December 2, 2018
}

\begin{abstract}
Sharp edges in X-ray surface brightness with continuous gas pressure called cold fronts have been often found in relaxed galaxy clusters such as Abell 496. Models that explain cold fronts as surviving cores of head-on subcluster mergers do not work well for these clusters and competing models involving gas sloshing have been recently proposed. Here, we test some concrete predictions of these models in a combined analysis of density, temperature, metal abundances and abundance ratios in a deep Chandra exposure of Abell 496. We confirm that the chemical discontinuities found in this cluster are not consistent with a core merger remnant scenario. However, we find chemical gradients across a spiral "arm" discovered at $73 \mathrm{kpc}$ north of the cluster center and coincident with the sharp edge of the main cold front in the cluster. Despite the overall SN Ia iron mass fraction dominance found within the cooling radius of this cluster, the metal enrichment along the arm, determined from silicon and iron abundances, is consistent with a lower SN Ia iron mass fraction $(51 \% \pm 14 \%)$ than that measured in the surrounding regions $(85 \% \pm 14 \%)$. The "arm" is also significantly colder than the surroundings by $0.5-1.6 \mathrm{keV}$. The arm extends from a boxy colder region surrounding the center of the cluster, where two other cold fronts are found. This cold arm is a prediction of current high resolution numerical simulations as a result of an off-center encounter with a less massive pure dark matter halo and we suggest that the cold fronts in A496 provide the first clear corroboration of such model, where the closest encounter happened $\sim 0.5$ Gyr ago. We also argue for a possible candidate dark matter halo responsible for the cold fronts in the outskirts of A496.
\end{abstract}

Subject headings: galaxies: clusters: individual (Abell 496) — intergalactic medium — cooling flows — X-rays: galaxies: clusters-

\section{INTRODUCTION}

One of the most interesting features discovered by Chandra satellite observations of galaxy clusters are the sharp X-ray surface brightness discontinuities, accompanied by jumps in gas temperature named "cold fronts" (e.g. Markevitch et al. 2000; Vikhlinin et al. 2001; Mazzotta et al. 2001). The temperature and density jumps happen in such a way as to maintain the gas pressure continuously across the front and, therefore, they are not created by shocks. They were originally interpreted as being the result of subsonic (transonic) motions of head-on merging substructures with suppressed thermal conduction (Markevitch et al. 2000, 2001; Vikhlinin et al. 2001).

The above mentioned merger core remnant model is theoretically justified (e.g. Bialek, Evrard \& Mohr 2002; Nagai \& Kravtsov 2003; Heinz et al. 2003; Mathis, et al. 2005; Poole et al. 2006) and holds relatively well for clusters that have clear signs of merging, such as 1E0657-56 (Markevitch et al. 2002) and A3667 (Vikhlinin et al. 2001). However, these models do not work well for the increasing number of cold fronts (sometimes multiple cold fronts in the same cluster) found in apparently non-merging clusters such as A496 (Dupke \& White 2003, hereafter DW03), A1795 (Markevitch et al. 2001), RXJ1720.1+2638 (Mazzotta et al. 2001). This prompted the development of other models for cold front genera- tion, such as oscillation of the $\mathrm{cD}$ and the low entropy gas around the bottom of the potential well (Lufkin et al. 1995; Fabian et al. 2001; DW03), hydrodynamic gas sloshing (Ascasibar \& Markevitch 2006, hereafter AM06), or dark matter peak oscillation due to scattering of a smaller dark matter system (Tittley \& Henriksen 2005). For very recent review see Markevitch \& Vikhlinin (2007).

Cold fronts are found with relatively high frequency. A review of Chandra archival images finds that more than $40 \%$ of the observed clusters have cold front-like features and their presence may have significant physical impact in the physics of their host cluster cores, such as gas heating, generation of bulk and turbulent velocities, constraining conduction, etc. The significance of cold fronts influence on cluster physics depends on how they are being generated. Therefore, it is important to determine which mechanisms actually produce cold fronts. Abell 496 provides an excellent opportunity to test different scenarios for cold front generation given its physical and observational characteristics. A496 is a typical, bright, nearby ( $\mathrm{z} \approx 0.032$ ), apparently relaxed cold core cluster. The Xray peak coincides very well with the $\mathrm{cD}$ optical centroid. The gas temperature varies from $5-6 \mathrm{keV}$ in the outer regions to $2-3 \mathrm{keV}$ in the central arcmin (e.g. Tamura et al. 2001, DW03). The presence of a central abundance enhancement has been established with previous instruments including Ginga and Einstein (White et al. 1994), 
ASCA (e.g. Dupke \& White 2000a), BeppoSAX (Irwin \& Bregman 2001) and XMM (Tamura et al 2001), showing an overall radial enhancement from $\sim 0.2-0.25$ solar in the outer regions to $\sim 0.4-0.7$ solar in the central arcmin. Furthermore, Dupke \& White (2000a) also discovered radial gradients, for the first time, in various elemental abundance ratios, which indicates that the gas in the central $2-3^{\prime}$ has a higher proportion of SN Ia ejecta $(\sim 70 \%)$ than the outer parts of the cluster. This was confirmed by more sensitive spectrometers on-board XMM (Tamura et al 2001).

As pointed out by DW03, different models for cold front formation can be discriminated through the analysis of chemical gradients across the front. If the cold front is a due to a head-on merger core remnant, we should expect the front to be accompanied by a specific discontinuity of elemental abundance ratios (e.g. Mushotzky et al. 1996; Dupke \& White 2000a,b). The expected discontinuity in this case would be symmetric with respect to the merger axis and asymmetric with respect to the perpendicular direction to the merger axis. This kind of analysis can be performed best with Chandra, given its high angular resolution. DW03 performed a chemical analysis of the cold front in Abell 496. With an effective exposure of $\sim 9 \mathrm{ksec}$, they were able to determine abundance ratio profiles only on large semi-annuli, covering a region larger than that of the cold front itself. The distribution of iron, silicon and oxygen abundances showed radial gradients but there were no clear discontinuities uniquely related to the cold front itself, pointing out the weaknesses of the remnant merging core model when applied to A496. Here we report the results of a deeper observation of that cluster that allowed us to produce high quality maps of the gas parameters and to compare more closely the observations with the predictions given by different models for cold front formation. All distances shown in this Letter are calculated assuming a $\mathrm{H}_{0}=70 \mathrm{~km} \mathrm{~s}^{-1} \mathrm{Mpc}^{-1}$ and $\Omega_{0}=1$ unless stated otherwise. At the distance of this cluster $1^{\prime \prime} \approx 0.66 \mathrm{kpc}$.

\section{DATA REDUCTION}

Abell 496 was observed by Chandra ACIS-S3 in July 2004 for $76 \mathrm{ksec}$. The cluster was centered on the S3 chip. We used Ciao 3.2.0 with CALDB 3.0 to screen the data. After correcting for a short flare-like period the resulting exposure time in our analysis was 59.6 ksec. A gain map correction was applied together with PHA and pixel randomization. ACIS particle background was cleaned as prescribed for VFAINT mode. Point sources were extracted and the background used in spectral fits was generated from blank-sky observations using the acis_bkgrnd_lookup script. Here we show the results of spectral fittings with XSPEC V11.3.1 (Arnaud 1996) using the apec and Vapec thermal emission models. Metal abundances are measured relative to the solar photospheric values of Anders \& Grevesse (1989). Galactic photoelectric absorption was incorporated using the wabs model (Morrison \& McCammon 1983). Spectral channels were grouped to have at least 20 counts/channel. Energy ranges were restricted to $0.5-9.5 \mathrm{keV}$. The spectral fitting errors are 1- $\sigma$ confidence unless stated otherwise.

In order to obtain an overall distribution of the spectral parameters we used an adaptive smoothing code that selects extraction regions based on a fixed minimum number of counts per cell (here we used 3000 counts for temperatures and global abundances and 7000 for individual abundances) to maintain the range of statistical fitting errors more or less constant throughout. The intercell spacing is fixed at a fraction of the radius of the surrounding cells and in general there is significant cell to cell overlap except for the cells with smallest size. The overlap of extraction regions is therefore stronger in low surface brightness regions, away from the core of the cluster. We plot the distribution of region sizes in Figures 3e and 4e, to give an estimate of the local smoothing kernel size. The code produces a matrix with best-fit values and different cell sizes. The best-fit values used here are defined as the mid point of the $68 \%$ confidence errors. In order to make the contour plots, this matrix is mapped into a square matrix with equal cell sizes using an interpolation routine. This is done by computing a new value for each cell in the regular matrix weighing by the values of the adjoining cells in the matrix included within some defined search radius (minimum of 3 cells in 4 adjacent quadrants). The closest measured values usually have the most influence on calculating the value of a cell. The computation is based on the Kriging method (for a description see, e. g., Davis, 1986, p.383), which calculates the weights from a semivariogram $\left(\gamma(h)=\frac{\sum_{i}\left(X_{i}-X_{i+h}\right)^{2}}{2 n}\right)$ developed from the spatial structure of the data, where $h$ is the number of intervals between the values of the regionalized variable $X$ taken at location $i$ and $i+h$ and $n$ is te total number of points. The number of cells of the mapped matrix was artificially increased to three times of the maximum length of the original matrix for purposes of improving image quality for analysis. This is responsible for the small "square domains" that appear in Figures 3a,c \& 4a,c. The values outside the CCD border contours are also an effect of the smoothing algorithm and should be ignored.

\section{RESULTS}

\subsection{Cold Fronts and Temperature Distribution}

Figure 1a shows the exposure corrected smoothed Xray image of A496. One can clearly see the sharp surface brightness edge towards the north, described in DW03. One can also see two other brightness edges (to the SW and SE) that meet at nearly right angles. This suggests the presence of multiple cold fronts in this cluster ${ }^{1}$. To analyze the nature of these edges we used the set of extraction regions shown in Figure 1b. The results are shown in Figures 2a,b using a wabs apec spectral model. Figure 2a shows the distribution of surface brightness (top) and projected gas temperatures using the bins shown in Figure $1 \mathrm{~b}$ (bottom) . The color association between the Figures 2 and Figure 1b is: North-black, East-red, South-blue and West-green. The locations of the cold fronts are marked by vertical dashed lines and follow the same color code. There are at least three (up to five) surface brightness edges accompanied by sudden temperature jumps, consistent with cold fronts; The northern one is at $\sim 73 \mathrm{kpc}$ and is the strongest. The western cold front is nearly at the same radial distance $(\mathrm{r} \sim 64 \mathrm{kpc})$ as the northern one

${ }^{1}$ Tanaka et al. (2006) finds an additional cold front in this cluster $4^{\prime}$ to the south, out of the field of view of our observation 
and is, apparently, an extension of the northern front. We can also see that the two edges near the core, labeled East $(\mathrm{r} \sim 16 \mathrm{kpc})$ and South $(\mathrm{r} \sim 22 \mathrm{kpc})$ have the temperature jumps characteristic of cold fronts. There is also a marginally significant cold front to the east at $\mathrm{r} \sim 106 \mathrm{kpc}$.

Following DW03, we measured the radial distribution of metal abundance ratios towards the directions of the main fronts (edges). We used "PIE" extraction regions that were chosen in such a way as to have the same opening angle as the cold front of interest. In the radial distributions there are no clear significant systematic relations between the changes in Fe abundance or abundance ratios and cold fronts. The changes seen can be mostly associated with overall (global) radial trends. Globally, the $\mathrm{Fe}$ abundance shows a radial decline from supersolar near the cluster's center to subsolar in the outer core regions. At the very center, $\mathrm{r} \leqslant 10^{\prime \prime}(\sim 7 \mathrm{kpc})$, there is a significant abundance dip described in the next section. The radially average values in the central $23 \mathrm{kpc}$ is $0.93 \pm 0.04$ solar (with asymmetric variations from 0.8 solar to 1.2 solar) and in the outer $(130 \pm 50) \mathrm{kpc}$ is $0.75 \pm 0.04$ (with asymmetric variations from 0.47 solar to 0.86 solar).

The results for the ratios involving $\mathrm{Si}, \mathrm{S}$, and $\mathrm{Fe}$ are shown in Figure 2b, where the color code is the same as that used in Figure 2a. In the abundance ratio plots we added "2" to the values of the Northern and Western directions, for illustration purposes. Despite the strong anisotropies, particularly in the bins within $\sim 70-110 \mathrm{kpc}$, there is also a tendency for the $\alpha$-element ratios to Fe grow radially. The $\mathrm{Si} / \mathrm{Fe}$ abundance ratio is consistent with a flat (or mildly increasing) profiles going from $1.39 \pm 0.10$ in the central $\sim 11 \mathrm{kpc}$ to $1.52 \pm 0.23$ in the very outer core regions $(\gtrsim 110 \mathrm{kpc})$. In the same regions, the $\mathrm{S} / \mathrm{Fe}$ ratio exhibits a more significant gradient, growing from $\sim$ $1.46 \pm 0.14$ solar to $2.34 \pm 0.38$ solar. The error weighted average of the SN Type dominance of the Fe mass from the two ratios above corresponds to $65 \% \pm 4 \% \mathrm{SN}$ Ia Fe mass fraction for the central region and $57 \% \pm 7 \%$ in the outer regions. This is consistent with the general trend found with ASCA by Dupke \& White (2001a) for larger spatial scales (up to $\lesssim 500 \mathrm{kpc}$, although the absolute values of the sulfur abundance are higher than those determined with ASCA (Dupke \& White 2000a) and XMM (Tamura et al. 2001) ${ }^{2}$.

The variations in the radial distributions of abundance ratios and temperatures suggest the presence of significant asymmetries. To explore the nature of these asymmetries we produced 2-dimensional adaptively smoothed maps of projected gas temperatures, abundances and abundance ratios. We discuss them in the next section.

\subsection{2-D Maps}

Given the level of asymmetry of the distributions of gas temperature, metal abundances and abundance ratios, it is helpful to analyze the 2-D distributions of these parameters. The temperature and $\mathrm{Fe}$ abundance maps are shown in Figures 3a,c, with X-ray surface brightness contours used in Figure 1b overlaid. The steepest temperature gradient is seen to the North. One striking feature that can be seen in the temperature map is a "cold spiral arm" that departs from the core to the N-NW up to the cold front position and runs along the cold front to the E-NE becoming more diffuse as it turns towards the S. The smoothing kernel radius map (Figure 3e) shows a value of $\sim 20(30)$ pix or $10^{\prime \prime}\left(15^{\prime \prime}\right)$ in the inner (outer) arm regions, which is nearly half of the arm thickness and indicates that the arm is well spatially resolved. Guided by the temperature map, we defined regions that characterize the inner and outer parts of the arm for spectral extraction and they are shown in Figures 3a,c \& 4a,c, and the relevant best fit parameters are shown in Table 1 . The temperature of the cold arm is $\sim 3.08 \pm 0.07 \mathrm{keV}$. The temperatures on the surrounding regions of the cold arm are $3.5 \pm 0.11 \mathrm{keV}$ and $4.7 \pm 0.22 \mathrm{keV}$ towards the inner and outer cluster regions, respectively. The cold arm is definitely associated with the northern cold front and to a lesser extent to the western cold front. It departs from a boxy low temperature region, the edges of which appear coincide with the southeastern and southern cold fronts near the cluster's core, although the temperature edges in these weaker cold fronts are less well-defined than that of the main cold front. From Figure $3 \mathrm{~b}$ it can be seen that the overall temperature error in the cold arm region is around $0.1-0.2 \mathrm{keV}$. The higher temperatures near the southern CCD border are not well constrained (with errors $\gtrsim 1 \mathrm{keV}$ ).

There are significant indications of a "cold tail" ( $\mathrm{T} \sim$ $4 \mathrm{keV}$ ) starting $2^{\prime} .3$ southwest of the cluster's center extending to $4^{\prime} .2$ to the south of the cluster that is associated with a low $\mathrm{Fe}$ abundance region (Figure 3c). The abundance along the cold tail is approximately half of the surrounding regions values of $\sim 1.2$ solar. This "cold tail" seems to extend to the south for more $5^{\prime}$ (Tanaka et al. 2006). A similar cold tail was found on the opposite side of the cold front in the cluster 2A0335+096 (Tanaka et al. 2006).

The Fe abundance map is also inhomogeneous (Figures $3 \mathrm{c}, \mathrm{d})$. There is an overall abundance gradient, which is steeper towards the northern regions. In particular the transition from sub to super solar abundances happens at a radius of $100^{\prime \prime}-140^{\prime \prime}$ from the center in all directions but the South. In general, the Fe abundance within the main cold front spatial scales $(\mathrm{r}<60 \mathrm{kpc})$ is supersolar, with the exception of the very central $8 \mathrm{kpc}$, where an abundance "dip" is found. The Fe abundance in the central dip reaches a minimum of $0.55 \pm 0.3$ solar (an average $0.8 \pm 0.03$ solar in a circular region $10.5 \mathrm{kpc}$ in radius) and in the immediately surrounding regions achieves a maximum of $\sim 1.7 \pm 0.4$ solar (an average of $1.1 \pm 0.04$ solar within an annulus with radius between $11 \mathrm{kpc}$ and $22 \mathrm{kpc}$ ). There is a secondary, marginally significant, abundance dip with similar spatial scales $35^{\prime \prime}$ to the N-NW, where the abundance decreases from $\sim 1.3$ to $\sim 0.7$ solar with a characteristic error of 0.3 solar. Central metal abundance dips have been found in other clusters (e.g., A2199 (Johnstone et al. 2002), Centaurus (Sanders \& Fabian 2002) and Perseus (Schmidt et al. 2002)), and the mechanisms that gener-

\footnotetext{
${ }^{2}$ It should be noted that even though the radial trends found for ratios that include $\mathrm{S}$ are, in general, similar to those derived from other ratios, the absolute values of the $\mathrm{S}$ abundance seem to be overestimated with respect to SN Ia and II yield models. To place it within the theoretical models the values measured would need a systematic negative correction. This does not change the conclusions of this paper since we are looking at relative chemical changes across cold fronts. For a discussion about the discrepancies found between observed sulfur yields and model predictions see ,e.g., Dupke \& White 2000a; Baumgartner et al. 2005 and references therein)
} 
ate them are a matter of current debate. Suggested scenarios include resonant scattering (cf. Sanders \& Fabian 2006), extremely inhomogeneous metal abundances (Morris \& Fabian 2003), artifacts appearing from fitting single temperature models to multi temperature gas (Buote 2000) and buoyant transport to higher radii (Brighenti \& Mathews 2005). None of these mechanisms are adequate to explain off-center abundance dips, which are probably related to previous AGN activity. A extended analysis of off-center abundance dips in clusters is provided elsewhere (Dupke, Nyland \& Bregman 2007, in preparation). Metal abundances are in general high towards the southern regions, with the exception of the regions coincident with the southern cold tail.

We performed an analysis of the 2-D distribution of the elemental abundance ratios in this cluster. Different metal enrichment mechanisms act with different efficiencies at different cluster locations and produce different SN type ejecta signatures. Therefore, elemental abundance ratios can be used as "fingerprints" used to trace the gas history, better than metal abundances alone. The abundance ratio maps involving the best determined abundances ( $\mathrm{Si}, \mathrm{S}$, and Fe) are shown in Figures 4a, c. The 1- $\sigma$ errors of the quantities are shown in Figures $4 \mathrm{~b}$, d, and give an idea of the significance level of the measured quantity in the region of interest. Since our best-fit values are defined as the mid-point of the 1- $\sigma$ error bars, we use only values with fractional errors smaller than $100 \%$ were used to create the 2-D square images. This is done to avoid biases in the interpolation to produce the smoothed color contours that would be caused by upper/lower limits, where the error bars can be highly asymmetrical. It can be seen that, in general, the cold arm is accompanied by enhanced abundance ratio values (lower SN Ia Fe mass fraction than the surroundings), which is visible in the $\mathrm{Si} / \mathrm{Fe}$, which shows an average variation from $\sim 1$ to 2 , or equivalently, from $85 \%$ to $51 \%$ SN Ia Fe Mass fraction, respectively in the regions surrounding the cold arm and the regions along the cold arm. The characteristic error is $\sim 0.4(\sim 14 \%$ in SN Ia Fe mass fraction) and the characteristic smoothing kernel size is $\sim 30(50)$ pixels, or $15^{\prime \prime}\left(25^{\prime \prime}\right)$. Sulfur abundances are higher than expected and abundance ratios are off-scale when compared to the theoretical predictions of Nomoto et al. 1997a,b for SN Ia and II yields. However, the trend of $\mathrm{S} / \mathrm{Fe}$ is similar to that of $\mathrm{Si} / \mathrm{Fe}$ and to place the limits within theoretical bounds, we need to apply constant positive correction of $\sim 0.4$ to $\mathrm{S} / \mathrm{Fe}$, placing the and the corresponding $\mathrm{S}$ negative correction $\sim 0.4-0.8$ within the errors (see footnote 2).

\section{DISCUSSION: THE NATURE OF COLD FRONTS IN ABELL 496}

The analysis of the core of A496 presented in this Letter reveals several new features that were not observed previously. A large multiplicity of cold-front features (at least three cold fronts); a spiral cold arm seen in the temperature map, which is clearly associated with the main (northern) cold front; strong indication of spiral (or circular) chemical arms associated with the main cold front; a cold, metal poor tail extending towards the direction opposite to the main cold front; an overall central abundance enhancement with a small-scale "dip" at the core, and marginal evidence for other off-center abundance dips.
The multiplicity of cold fronts together with the spiral pattern of the chemical gradients seem to rule out the scenario, where the cold front(s) in this cluster are created by a head-on merging remnant core.

Although gas sloshing has been invoked to explain cold fronts in apparently relaxed clusters, there have been very few observable predictions that can be used to discriminate the details of different sloshing mechanisms proposed in the literature. Very recently, AM06 performed high resolution numerical + hydrodynamical simulations specifically designed to investigate the effects of scattering of lower mass dark matter haloes (with and without gas) by clusters of galaxies. One of the results from their work was that the sub-halo flyby induces a variable gas velocity field in the ICM of the main cluster that generates rampressure near the cluster gas core and produces cold fronts, accompanied by significant amount of substructures seen in the gas 2-D temperature distribution.

A common feature in most cases analyzed by AM06 was the presence of cold spiral arms coinciding with the cold fronts close to the main cluster's core, which were long lasting. In particular, their case for a dark matter perturber produces properties very similar to those observed in A496. In AM06 a pure dark matter halo with $\frac{1}{5}$ of the mass of the main cluster flies by with an impact parameter of $500 \mathrm{kpc}$ and with closest approach at t 1.37 Gyr. We show part of Figure 7 of AM06, for the epoch corresponding to 1.9 Gyr (Figure 5a). The image is inverted vertically to be compared directly to the temperature map of A496 in Figure 2a. The size of the box is $250 \mathrm{kpc}$, similar to the size of ACIS-S3 CCD borders at the redshift of the cluster $(\sim 320 \mathrm{kpc})$. The cold front(s) can be seen when comparing the temperature map with the surface brightness map (Figure 21 of AM06). The main cold front coincides with the large spiral cold arm extending horizontally. The spatial scale is very similar to that of the cold arm in A496. Their simulations also seem to indicate the presence of milder cold fronts in the opposite side closer to cluster's core. These are clear predictions that are corroborated well by A496 and suggest strongly that a flyby dark matter halo created the cold fronts in this cluster. Furthermore, there is a larger-scale more diffuse cold extension of the main arm also towards the South of the main cold front, which is a consequence of the ram-pressure caused by the gas velocity field induced by the DM halo flyby. This suggests that the same process that creates the main cold front may also be associated with the formation of the southern cold tail seen in A496. The existence of such pure DM sub-halos is not completely unexpected since the intergalactic gas originally belonging to the sub-halo could have been stripped in a previous encounter with the main cluster. AM06 cases for gaseous DM sub-clump passages produces a variety of substructures visible in temperatures and surface brightness maps, which are not seen in A496, and are not favored within the limited cases simulated. Future addition of metallicity distributions to cluster merger simulations should constrain further the characteristics of the perturber.

A prediction of this scenario is the presence of a DM halo in the outskirts of the cluster without significant Xray emitting gas. From the simulations, the position of that clump at epoch ( $t=2$ Gyr, i.e., now) would be towards 
its apocenter at North, the same general direction of the main cold front. It is reasonable to assume that galaxies would tend to trace their host DM sub-halo. Recent wavelet analysis of the member galaxies of A496 within a $1.5 \mathrm{~h}_{75}^{-1}$ Mpc radius (Flin \& Krywult 2006), finds a secondary galaxy clump, in most wavelet scales analyzed, to the NW of the core of A496, roughly consistent with the position where the DM perturber was likely to be found in the AM 06 simulation (towards the North). We illustrate this in Figure 5b, where we show the positions of the dark matter clump at 1.34, 1.43, 1.51 and 4.2 Gyr, taken from a merging 4 of the 9 images of Figure 3 of AM06. We overlap part of Figure 5 of Flin \& Krywult (2006), which illustrates the position of the galaxy sub-clump for a wavelet scale of $129 \mathrm{~h}_{75}^{-1} \mathrm{kpc}$.

If we scale the ratio of masses of the main cluster to hat of the DM perturber from the AM06 simulation parameters and, conservatively, use for A496 the mass of $4.2 \times 10^{14} \mathrm{M}_{\odot}$ (Durret et al. 2000), the perturber should be very massive $\left(0.84 \times 10^{14} \mathrm{M}_{\odot}\right)$. This is almost three times more massive than HCG62 (Morita et al. 2006), the brightest HCG in the Ponman et al. (1996) survey. Such a group, if not unusually depleted of gas, would easily be detected by current X-ray instruments at the A496 redshift. ROSAT All Sky Survey exposures of that region $\left(\mathrm{R}<50^{\prime}\right.$ from A496) fails to detect a significant X-ray excess from any extended sources as expected by the gasless dark matter perturber scenario described here. The excess count in a square region $18^{\prime}$ on the side centered in Flin \& Krywult's (2006) sub clump is $15 \pm 18$ background subtracted counts. However RASS exposures are too short $(\sim 250 \mathrm{sec})$ to place any significant constraints on the amount of $\mathrm{X}$ ray emitting gas and future combination of weak lensing and deeper X-ray observations of that substructure with current satellites should be able to corroborate this prediction.

We acknowledge support from NASA through Chandra award number GO 4-5145X, NNG04GH85G and GO56139X. RAD was also partially supported by NASA grant NAG 5-3247. RAD also thanks Yago Ascasibar, Jimmy Irwin, Tatiana F. Lagana, Narciso Benitez \& Tracy Clarke for helpful discussions.

\section{REFERENCES}

Anders, E. \& Grevesse N. 1989, Geochimica et Cosmochimica Acta, 53, 197

Arnaud, K. A. 1996, in Astronomical Data Analysis Software and Systems V, ASP Conf. Series volume 101, eds. Jacoby, G. \& Barnes, J., p.17

Ascasibar, Y. \& Markevitch, M., 2006, ApJ, in Press, astro-ph 0603246

Baumgartner, W. H., Loewenstein, M., Horner, D. J., \& Mushotzky, R. F. 2005, ApJ, 620, 680

Bialek, J. J., Evrard, A.E. \& Mohr, J.J., 2002, ApJ, 578, 9

Buote D. A., 2000, ApJ, 539, 172

Brighenti, F. \& Mathews, W., 2005, ApJ, 630, 864

Davis, J. C., 1986. Statistics and Data Analysis in Geology. John Wiley \& Sons, Inc. Second edition,

Dupke, R. A. \& White, R. E. III 2000a, ApJ, 537, 123

Dupke, R. A. \& White, R. E. III 2000b, ApJ, 528, 139

Dupke, R. A. \& White, R. E. III, 2003, ApJ, 583, L13

Durret, F., Adami, C., Gerbal, D. \& Pisllar, V. 2000, å, 356, 815

Fabian, A. C. et al. 1981, MNRAS196, 35

Fabian, A. C., Sanders, J. S., Ettori, S., Taylor, G. B., Allen, S. W., Crawford, C. S., Iwasawa, K., \& Johnstone, R. M. 2001, MNRAS321, 33

Fabian, A. C., Sanders, J. S., Taylor, G. B., \& Allen, S. W., 2005, MNRAS360, L20

Flin, P. \& Krywult, J 2006, å450, 9

Heinz, S., Churazov, E., Forman, W., Jones, C.\& Briel, U. G. 2003 , MNRAS, 346, 13

Helsdon, S. \& Ponman, T. J., 2000, MNRAS, 315, 356

Johnstone R. M., Allen S. W., Fabian A. C., Sanders J. S., 2002, MNRAS, 336, 299

Loewenstein, M. \& Mushotzky, R. F. 1996, ApJ, 466, 695

Lufkin, E. A., Balbus, S. A., \& Hawley, J. F. 1995, ApJ, 446, 529
Markevitch, M., \& Vikhlinin, A., 2007, Phys. Rep., 443, p.1

Markevitch M. et al. 2000, ApJ, 541, 542

Markevitch, M., Vikhlinin, A., \& Mazzotta, P. 2001, ApJ, 562, L153

Markevitch, M. et al. 2002, ApJ, 567, 27

Mathis, H., Lavaux, G., Diego, J. M., \& Silk, J. 2005, MNRAS, 357, 801

Mazzotta, P., Markevitch, M., Vikhlinin, A., Forman, W. R., David, L. P., \& VanSpeybroeck, L.2001, ApJ, 555, 205

Nagai, D. \& Kravtsov, A. V., 2003, ApJ, 587, 514

Morita, U. Ishisaki, Y., Yamasaki, N. Y., Ota, N., Kawano, N., Fukazawa, Y., \& Ohashi, T. 2006, PASJ, 58, 719

Morris R. G., Fabian A. C., 2003, MNRAS, 338, 824

Morrison, R. \& McCammon, D. 1983, ApJ, 270, 119

Mushotzky, R. F. et al. 1996, ApJ, 466, 686

Nomoto, K. et al. 1997a, Nuclear Physics A, Vol. A621, 467c

Nomoto, K. et al. 1997b, Nuclear Physics A, Vol. A616, 79

Poole, G. B., Fardal, M. A., Babul, A., McCarthy, I. G., Quinn, T., \& Wadsley, J. 2006, MNRAS, 373, 881

Ponman, T. J., Bourner, P. D. J., Ebeling, H., \& Bohringer, H. 1996 MNRAS, 283,690

Sanders, J. S. \& Fabian, A. C. 2002, MNRAS, 331, 273

Sanders, J. S. \& Fabian, A. C. 2006, MNRAS, 370, 63

Schmidt, R. W., Fabian, A. C., \& Sanders, J. S. 2002, MNRAS, 337, 71

Tanaka, T., Kunieda, H., Hudaverdi, M., Furuzawa, A., \& Tawara, Y., 2006, PASJ, 58, 703

Tamura, T. et al. 2001, A\&A, 379, 107

Tittley, E. R., \& Henriksen, M. 2003, ApJ, 563, 673

Vikhlinin, A., Markevitch, M., \& Murray, S. 2001, ApJ, 551, 160 White, R. E. III, et al. 1994, ApJ, 433, 583 
TABLE 1

Temperatures And Abundances Across the Cold Arma

\begin{tabular}{lcccccccc}
\hline \hline Region ${ }^{\mathrm{b}}$ & $\begin{array}{c}\text { Temperature } \\
(\mathrm{keV})\end{array}$ & $\begin{array}{c}\mathrm{Fe} \\
(\text { solar })\end{array}$ & $\begin{array}{c}\mathrm{Si} \\
(\text { solar })\end{array}$ & $\begin{array}{c}\mathrm{S} \\
(\text { solar })\end{array}$ & $\begin{array}{c}\mathrm{Si} / \mathrm{Fe} \\
\text { (ratio) }\end{array}$ & $\begin{array}{c}\mathrm{S} / \mathrm{Fe} \\
\text { (ratio) }\end{array}$ & $\begin{array}{c}\mathrm{Si} / \mathrm{S} \\
(\text { ratio) }\end{array}$ & $\begin{array}{c}\chi^{2} \\
\text { d.o.f. }\end{array}$ \\
\hline Inner & $3.55 \pm 0.11$ & $0.86 \pm 0.08$ & $0.88 \pm 0.23$ & $1.44 \pm 0.35$ & $1.03 \pm 0.29$ & $1.68 \pm 0.43$ & $0.61 \pm 0.22$ & $211 / 204$ \\
Arm & $3.08 \pm 0.07$ & $0.77 \pm 0.07$ & $1.60 \pm 0.20$ & $1.43 \pm 0.26$ & $2.08 \pm 0.32$ & $1.86 \pm 0.38$ & $1.12 \pm 0.25$ & $176 / 217$ \\
Outer & $4.68 \pm 0.22$ & $0.75 \pm 0.13$ & $0.72 \pm 0.57$ & $2.16 \pm 0.74$ & $0.96 \pm 0.78$ & $2.89 \pm 1.10$ & $0.29 \pm 0.24$ & $167 / 171$ \\
\hline
\end{tabular}

${ }^{\text {a }}$ From wabs Vapec model

${ }^{\mathrm{b}}$ Shown in Figures 3a,c \& 4a,c

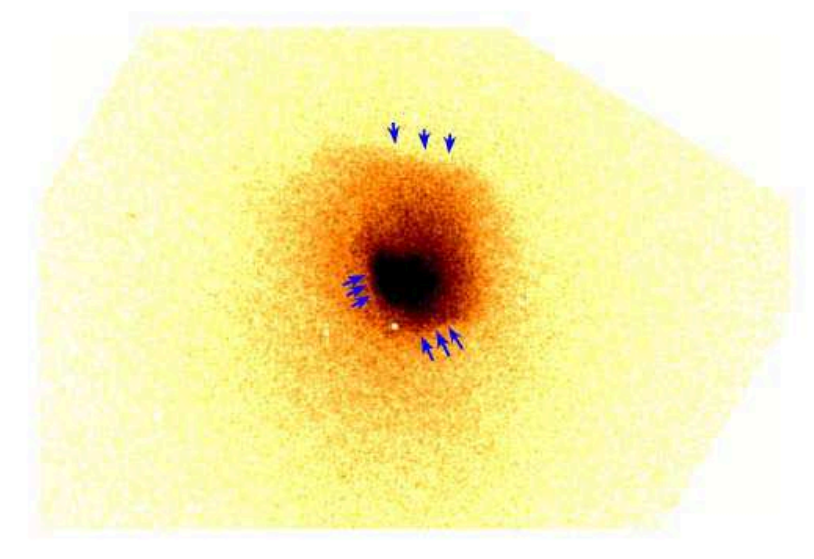

$2 \mathrm{~B}-07$

$6 \mathrm{E}-07$

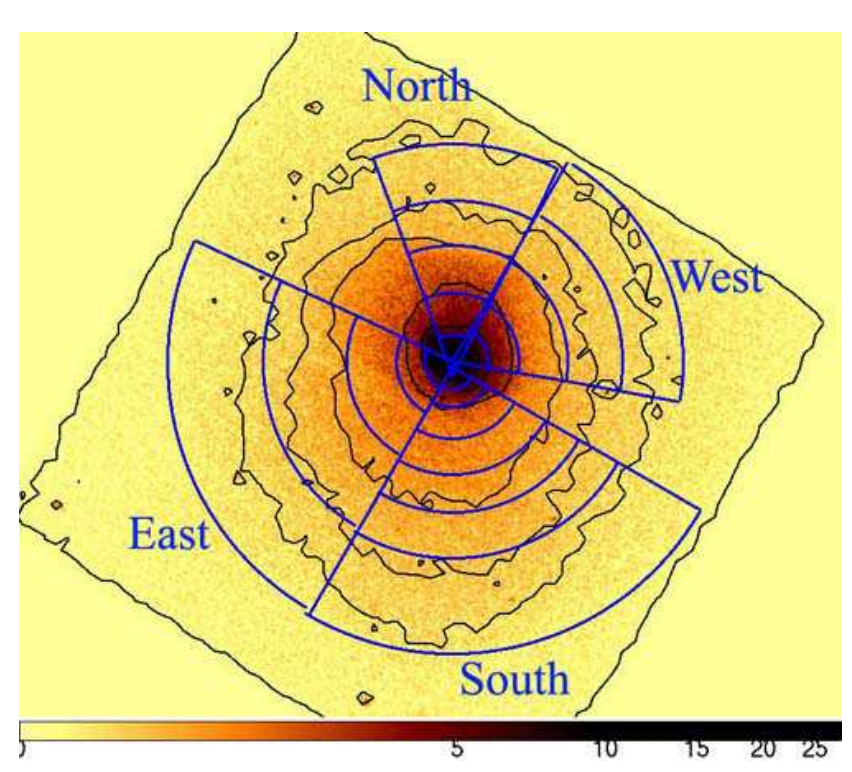

Fig. 1.- (a) Exposure corrected Chandra image of Abell 496 smoothed in DS9 version 4.0 with a Gaussian function with 3 pixel kernel radius. Blue arrows show the position of the northern, southern and eastern main cold fronts. (b) Same as (a0 but with the PIE sectorial extraction regions used in the radial analysis of the cold fronts. X-ray contours are also overlaid and are the same as in Figures 2 and 3.The outermost contour corresponds to ACIS-S3 chip border. 



Fig. 2.- (a)Temperature and Density Profiles: Exposure corrected surface brightness Top and best-fit gas temperature Bottom radial distributions along the line of symmetry of the sharp edge (cold fronts) indicated in Figure 1b, North (black), East (red), South (blue) and West (green) using a wabs apec spectral model. The vertical lines indicate the position of the cold fronts using the same notation for color as the data points. The extraction regions correspond to those shown in Figure $1 \mathrm{~b}$. The units are pixels and 1 pixel $=0.5^{\prime \prime} \approx 0.33 \mathrm{kpc}$. Errors are $1-\sigma$ confidence. Vertical lines indicate the position of the cold fronts. (b) Individual Iron and Metal Abundance Ratio Radial Distributions. Results from spectral fittings using a wabs Vapec spectral model of same regions as in (a) and the same color code. The values of $\mathrm{Si} / \mathrm{Fe}, \mathrm{S} / \mathrm{Fe}$ and $\mathrm{Si} / \mathrm{S}$ for the NORTH (black) and WEST (green) directions were added 2 to their original values for clarity. The reduced chi-squared for the spectral fittings shown are typically $0.7-0.96$, with typically $180-250$ degrees of freedom. 

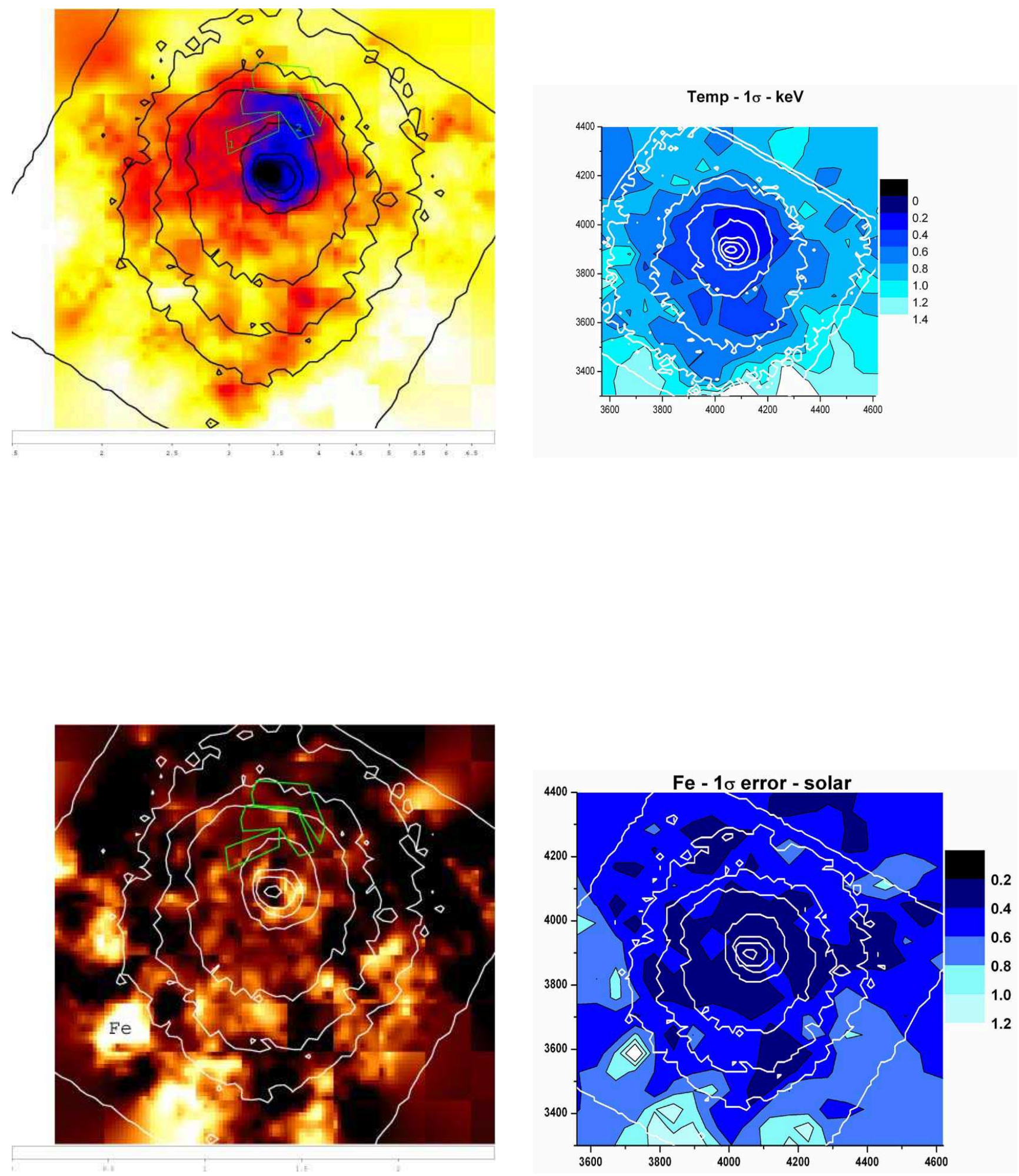
Spectral Extraction Region Radius (pixels $=0.5$ arcsec)

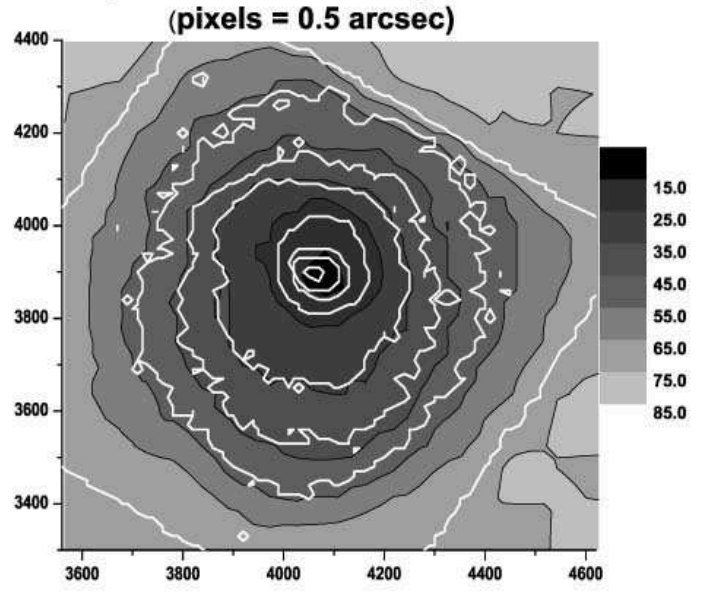

Fig. 3.- Results from an adaptive smoothing algorithm with a minimum of 3000 counts per extraction region (circular) and fitted with an absorbed VAPEC spectral model. The gridding method used is a correlation method that calculates a new value for each cell in the regular matrix from the values of the points in the adjoining cells that are included within the search radius, using the Kriging method (e.g. Davis 1986), see section 2 for details. We also overlay the X-ray contours shown in Figure $1 \mathrm{~b}$ on top). North is up. The units are pixels and 1 pixel $=0.5^{\prime \prime} \approx 0.33 \mathrm{kpc}$. The outermost contour corresponds to ACIS-S3 chip border and is centered at RA=68.4084 deg, Dec $=-13.261 \mathrm{deg}$. Values outside the CCD borders are an effect of the smoothing algorithm and should be ignored. The parameters mapped are (a) Temperature \& (c) Abundance. (b) and (d) show the adaptively smoothed 1- $\sigma$ error maps for temperatures and abundances, respectively. Figure (e) shows a color contour plot of the radii of the spectral extraction regions used to determine the parameters shown in (a)-(d). It basically gives an idea of the resolution, or smoothing kernel radius, of the 2-D maps above. 

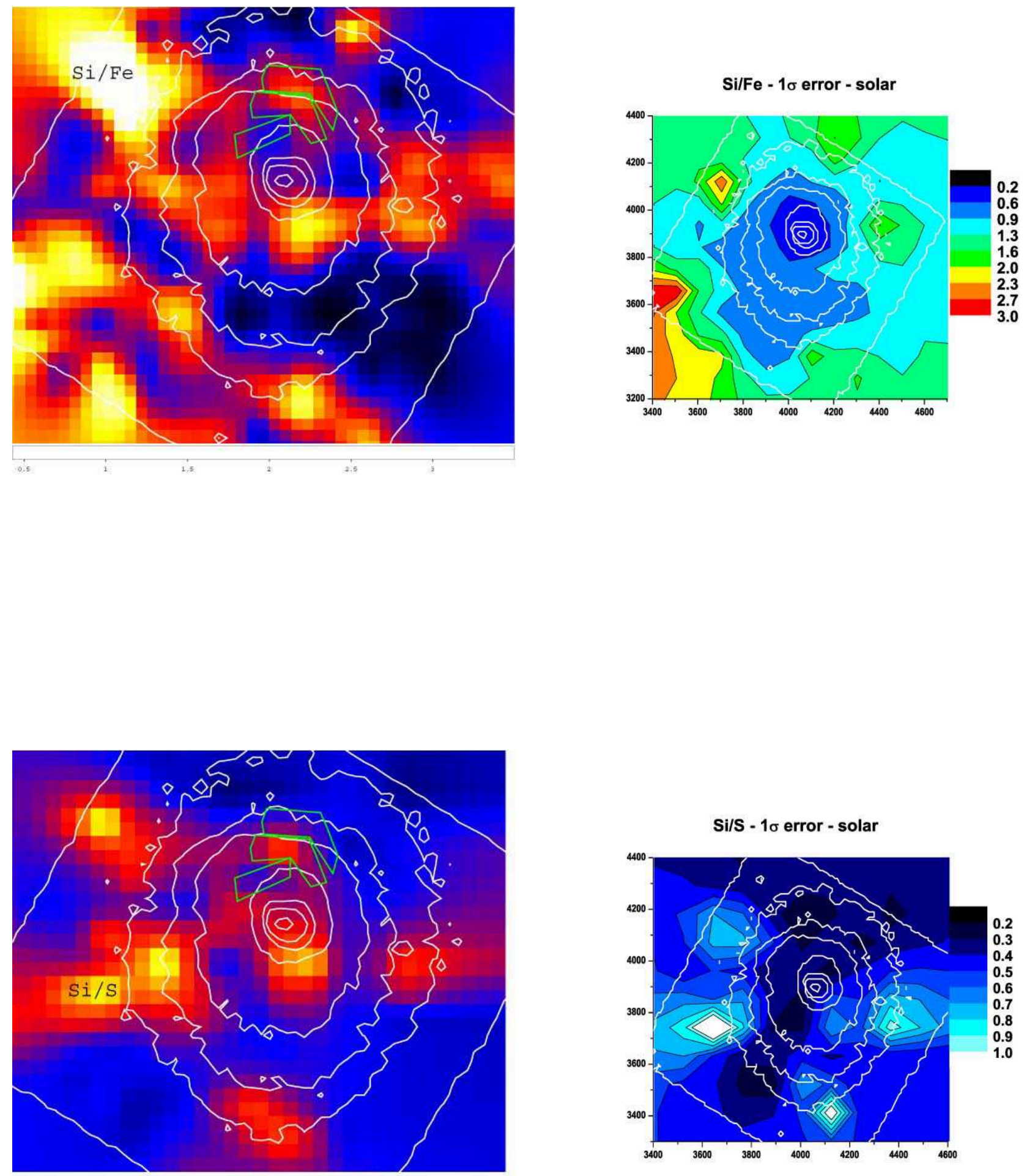




Fig. 4.- Individual Metal Abundance Ratios. Same notation as in Figures 3 but for the silicon to iron (a, b) and silicon to sulfur (c, d) ratios. (e) shows, analogously, the radii of the spectral extraction regions used to determine the parameters in Figures 3(a)-(d)
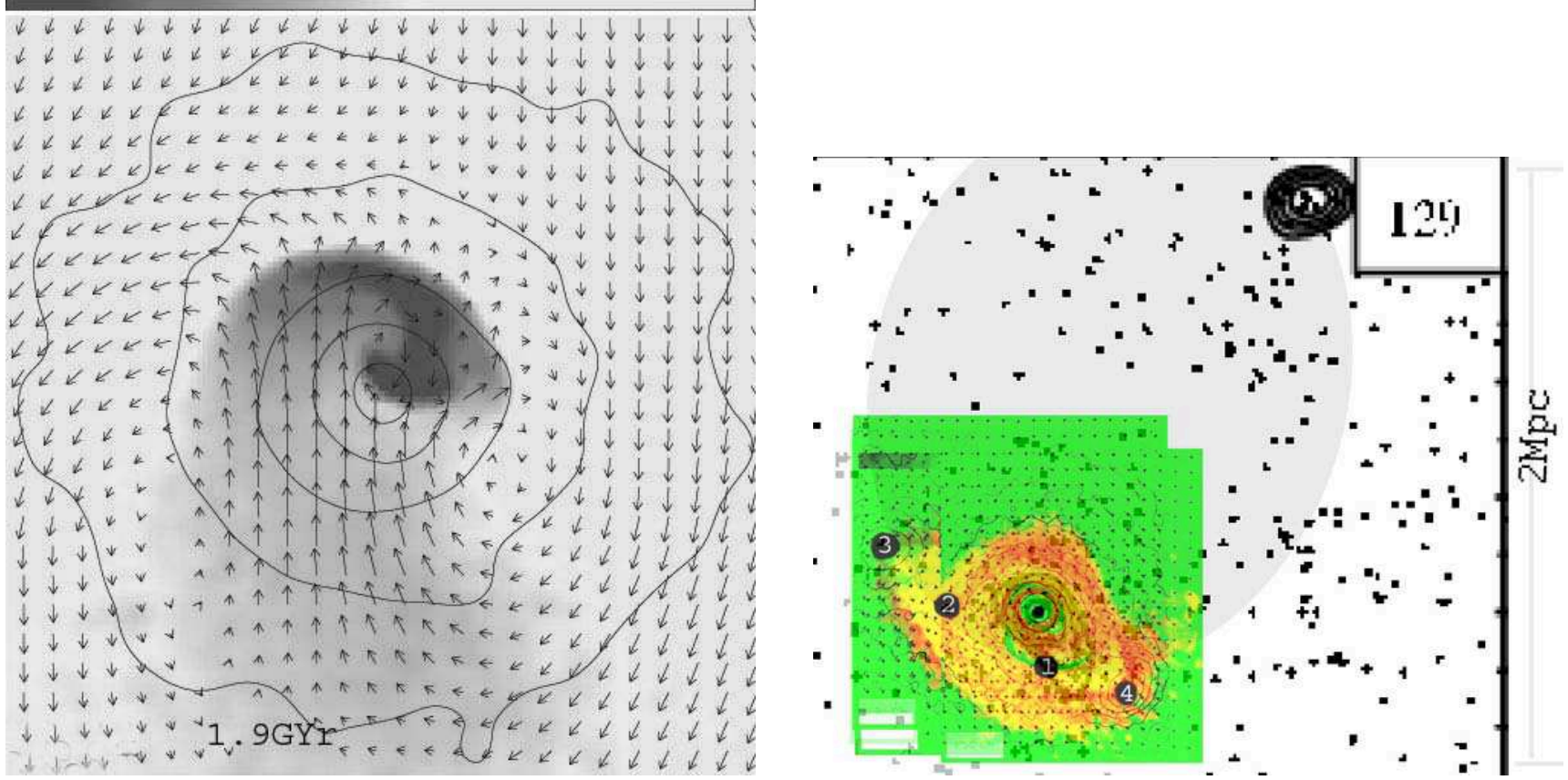

Fig. 5.- (a) zoom in of the core of simulated cluster 0.5 Gyr after the flyby of the dark matter halo. From AM06 (fig.7). Yellow is $\sim 7-9 \mathrm{keV}$ and blue $2 \mathrm{keV}$. DM density contours are overlaid and arrows indicate gas velocity (the longest corresponding to $500 \mathrm{~km} \mathrm{~s}^{-1}$. The size of the panel is $250 \mathrm{kpc}$. The figure has been flipped vertically to match the configuration of the cold front in A496. (b) Zoom out of the simulation in (a) for epochs 1.34 Gyr, 1.43 Gyr, 1.51 Gyr and 4.2 Gyr, indicated by numbers $1,2,3$ \& 4, respectively, merged and placed in scale to galaxy distribution map of Flin and Krywult (2006) with wavelet image for scale of 129 kpc shown by the contours. 